\title{
TEMA 7-2014: DERMATITIS AUTOINMUNE, URTICARIA GIGLICA/ANGIOEDEMA Y ANAFILAXIS POR PROGESTERONA
}

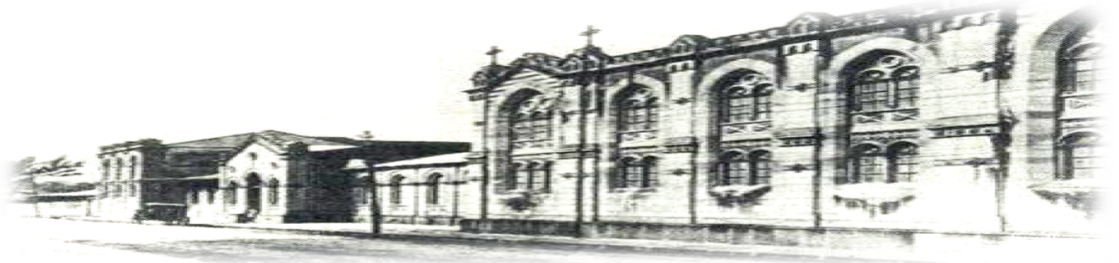

Haspital San quan de Dias. San Jasé. Costa Rica. Fundada en 1845

Recibido: $\quad$ 05/03/2014

Aceptado: $\quad 26 / 04 / 2014$

\author{
Melissa Ramírez Salas ${ }^{1}$ \\ Eduardo Fiedler Velásquez ${ }^{2}$
}

\begin{abstract}
${ }^{1}$ Médico Cirujano. Centro de Especialidades Médicas Alpha. Alajuela. Correo electrónico: dra.mramirez@medicos.cr

${ }^{2}$ Médico Especialista en Medicina Interna. Servicio de Medicina. Hospital Calderón Guardia. Profesor del posgrado en Medicina Interna UCR-CENDEISSS.
\end{abstract}

\section{RESUMEN}

Las reacciones cíclicas por progesterona son entidades clínicas muy poco comunes que describen aquellos episodios recurrentes $\mathrm{o}$ cíclicos de reacciones alérgicas multisistémicas, que ocurren alrededor de la menstruación y que al parecer, están relacionadas o son exacerbadas durante la fase lútea del ciclo menstrual. Hay poca información al respecto y se encuentran muy pocos reportes de casos que describan dichas reacciones ${ }^{(1,2)}$. Su diagnóstico es de exclusión y representa un reto considerable desde el punto de vista clínico y del manejo. Los puntos clave para su diagnóstico incluyen la aparición de los síntomas en la fase premenstrual, la desaparición de los mismos con la inhibición de la ovulación y una prueba cutánea positiva a la inyección intradérmica de progesterona. El pilar del tratamiento lo constituye la inhibición de la secreción endógena de progesterona, mediante la supresión de la ovulación con manejo médico, aunque en casos de pacientes con sintomatología severa y refractaria, puede ser necesaria la ooferectomía bilateral. La presente revisión tiene como objetivo actualizar los conocimientos que existen sobre las diferentes presentaciones de las reacciones de hipersensibilidad por progesterona, para poder brindar un diagnóstico certero y un tratamiento adecuado

\section{PALABRAS CLAVE}

Dermatitis autoinmune. Progesterona. Angioedema cíclico. Anafilaxis catamenial. Urticaria cíclica.

\section{ABSTRACT}

Cyclic progesterone reactions are rare clinical entities that describe recurring or cyclic episodes of multisystemic allergic reactions that occur around menses, and apparently are related or are exacerbated during the lutheal phase of the menstrual cycle. There is little information when 
searching the medical literature and very few case reports describing such reactions. It is an exclusion diagnosis that represents a considerable challenge from the clinical point of view and management. The key points for diagnosis include the onset of symptoms during the premenstrual phase, the disappearance with the inhibition of ovulation and a positive intradermal skin test with progesterone. The mainstay of treatment is the inhibition of secretion of endogenous progesterone by suppressing ovulation with medical treatment, although in certain cases with severe and refractory symptoms oophorectomy may be needed. The present review aims to update the concepts of the different and varied presentations of progesterone hypersensitivity reactions to provide an accurate diagnosis and appropriate treatment.

\section{KEY WORDS}

Autoimmune dermatitis. Progesterone. Cyclic angioedema. Catamenial anaphylaxis. Cyclic urticaria.

\section{INTRODUCCIÓN}

Se encuentra muy bien documentado cómo el ciclo menstrual tiene influencia en muchas mujeres en el desarrollo de enfermedades sistémicas, manifestaciones como acné o empeoramiento del acné, retención hídrica, porfiria, angioedema hereditario, úlceras aftosas, Síndrome de Behçet, epilepsia, migrañas, miastenia gravis ${ }^{(3)}$ y rinitis alérgica ${ }^{(4)}$.

Sin embargo, no se sabe bien aún cómo en pocas mujeres el ciclo menstrual puede inducir un espectro de condiciones alérgicas cíclicas, con una variedad de presentaciones en las que se incluyen urticaria, eczema, angioedema y aún, más raramente, síntomas de asma o anafilaxia ${ }^{(5)}$ que aparecen o son exacerbados cíclicamente en la fase lútea del ciclo $y$ resuelven total o parcialmente después de la menstruación ${ }^{(6)}$.

La progesterona es el agente etiológico más frecuentemente encontrado y es considerada como una reacción de hipersensibilidad a los niveles elevados de progesterona en la fase lútea del ciclo menstrual.

\section{DISCUSIÓN}

\section{Epidemiología}

El primer caso documentado de urticaria cíclica asociado a la menstruación fue descrito por Geber en $1921^{(7)}$. En el mismo, se identificó lo que podría corresponder a una alergia hormonal, ya que al practicar una sobrecarga con el propio suero premenstrual de una paciente, se observó que le causaba lesiones urticariformes agudas. Posteriormente, además, el mismo investigador utilizó la desensibilización con inyecciones sistemáticas del suero premenstrual de la paciente y observó cómo exitosamente podía ser usado para desensibilizar y disminuir los síntomas $^{(8)}$.

Desde entonces, solamente cerca de 50 casos dermatitis autoinmune por progesterona han sido publicados en la literatura médica, solamente 9 de anafilaxis inducida por progesterona $\mathrm{y}$ únicamente 2 casos de anafilaxis catamenial, lo que demuestra que los reportes de caso de este proceso son extremadamente raros.

La edad de inicio es variable, y en la literatura se ha reportado el caso de inicio más temprano empezando en la menarca ${ }^{(9)}$.

\section{Etiología}

La patogénesis exacta de este trastorno aún se desconoce, sin embargo se cree que está relacionada a una reacción de hipersensibilidad desencadenada por la progesterona.

La base autoinmune de la condición la sugieren las pruebas cutáneas positivas con progesterona o sus derivados, la reproducción de los síntomas con pruebas de sobrecarga hormonal intramuscular y la detección de anticuerpos contra la progesterona o sus derivados.

Los resultados obtenidos con las pruebas cutáneas con progesterona han mostrado reacciones inmediatas (menores a 30 minutos), tardías ( $24-48$ horas) y reacciones mixtas, tanto con características inmediatas como tardías ${ }^{(10)}$.

Las reacciones anafilácticas alrededor de la fecha de menstruación pueden ser tanto alérgicas como pseudo-alérgicas. Típicamente ocurre en mujeres debido a un fenómeno autoinmune a la producción de progesterona endógena, pero 
también puede ser causado por la administración exógena de una progestina sintética. En muchos casos, ocurre en mujeres que toman o han tomado anticonceptivos orales, los cuales pueden actuar como un desencadenante de la reacción ${ }^{(11,12)}$.

Como se mencionó anteriormente, el mecanismo involucrado no se ha entendido claramente, aunque la mayoría de los autores coinciden en una hipersensibilidad a la progesterona como causa subyacente ${ }^{(13)}$. Esta teoría fue apoyada con algunas pacientes quienes presentaron un resultado positivo cutáneo y sistémico a la prueba intradérmica con medroxiprogesterona. Sin embargo, en un reporte de cuatro pacientes con reacciones anafilácticas cíclicas similares, dos de las cuatro pacientes no mostraron positividad a la prueba cutánea ${ }^{(14)}$.

Otro mecanismo propuesto involucra un constituyente vasoactivo del flujo menstrual como lo son las prostaglandinas ${ }^{(2)}$. Esta teoría ha sido apoyada al encontrar que la $\mathrm{PGF}_{2}-\alpha$ juega un papel importante como modulador de la liberación de mediadores de los mastocitos ${ }^{(15)}$. Otra posibilidad es la participación de $\mathrm{PGI}_{2}$ (prostaciclina), que actúa como un vasodilatador potente que lleva a estas reacciones sistémicas en pacientes susceptibles, aunque de igual manera se han practicado pruebas cutáneas intradérmicas con flujo menstrual y no todas las pacientes presentan un resultado positivo ${ }^{(16)}$.

\section{Fisiopatología}

Probablemente existen varios mecanismos involucrados en el proceso que representa la hipersensibilidad autoinmune por progesterona, ya que en las pruebas cutáneas o intramusculares se obtienen diferentes tipos de reacciones, lo cual indica diferencias en la causas. Por ejemplo, algunas mujeres muestran reacciones inmediatas que ocurren minutos después de la prueba, otras son tardías y toman horas en aparecer los síntomas y además, existen otras en las que aparecen ambos tipos de reacciones.

Algunas posibilidades que se han descrito incluyen una estimulación de los linfocitos T-helper tipo 2 por la progesterona (lo que a su vez regula la síntesis de IgE y el proceso alérgico), efectos directos de la progesterona en los mastocitos y basófilos o la progesterona comportándose como un autoantígeno y produciendo respuestas de anticuerpo.

\section{Manifestaciones Clínicas}

Las reacciones alérgicas autoinmunes a la progesterona se manifiestan más comúnmente como lesiones cutáneas cíclicas en la piel. Las mujeres que presentan este trastorno en la mayoría de las ocasiones manifiestan erupciones dermatológicas en la fase lútea del ciclo menstrual y en algunos casos, se pueden presentar con compromiso de otros órganos además de la piel (ej. GI, Pulmón) lo que correspondería a una anafilaxis autoinmune por progesterona.

Los síntomas clínicos usualmente inician 3 a 10 días antes del inicio del flujo menstrual y finalizan 1 a 2 días después de la menstruación. La severidad de los síntomas puede variar desde ser casi indetectables hasta de naturaleza anafiláctica y además pueden ser progresivos ${ }^{(5,14)}$. Los síntomas autoinmunes por progesterona se correlacionan con los niveles de progesterona durante la fase lútea del ciclo menstrual ${ }^{(2)}$.

La gran variedad de síntomas como forma de presentación generalmente resulta en un diagnóstico tardío. En la literatura se han descrito lesiones eczematosas, urticaria, angioedema, erupciones similares a eritema multiforme, vesículas, lesiones bullosas o papulopustulares, manifestaciones similares a eritema anular centrífugo, eritema fijo medicamentoso y más aun, reacciones anafilácticas con urticaria, rubor facial, edema laríngeo, broncoespasmo, hipotensión $\mathrm{y}$ $\operatorname{shock}^{(13,14,18)}$.

La anafilaxis catamenial es otra entidad clínica que se diferencia de la dermatitis autoinmune por progesterona principalmente por el tiempo de aparición de los síntomas. La primera al parecer está directamente asociada con el comienzo del flujo menstrual y continúa hasta el final de la menstruación y en la segunda los síntomas inician entre 3 a 10 días antes de la menstruación y se mantienen hasta 1 a 2 días posterior al inicio del flujo menstrual ${ }^{(5)}$.

Dentro de algunas condiciones que pueden semejar una anafilaxis se pueden mencionar el síndrome carcinoide, feocromocitoma y mastocitosis sistémica ${ }^{(19)}$. 
Tabla 1. Dermatitis Autoinmune por Progesterona y sus diferentes manifestaciones.

\begin{tabular}{ll}
\hline $\begin{array}{c}\text { Reacciones } \\
\text { anafilactoides }\end{array}$ & \multicolumn{1}{c}{$\begin{array}{c}\text { Dermatológicas/Reacciones en } \\
\text { mucosas }\end{array}$} \\
\hline $\begin{array}{l}\text { Premenstrual } \\
\text { Catamenial }\end{array}$ & Estomatitis \\
& Eczema \\
& Eritema multiforme \\
& Síndrome de Stevens-Johnson \\
& Erupciones fijas por medicamentos \\
& Foliculitis \\
& Reacciones vesiculobulosas \\
& Urticaria \\
\hline Uuente: Adaptado de Snyder JL, et al. Dermatitis autoinmune por \\
progesterona y sus manifestaciones
\end{tabular}

\section{Diagnóstico}

Una vasta cantidad de reacciones alérgicas se pueden explicar debido al uso de diferentes medicamentos, dentro de los cuales podemos mencionar especialmente la aspirina y los antiinflamatorios no esteroideos, así como también pueden ser resultado dentro de otras causas de diferentes alimentos ${ }^{(20)}$. Debido a lo anterior, estas etiologías se deben tener en cuenta al evaluar a una paciente que presenta reacciones anafilácticas cíclicas y necesariamente deben ser descartadas antes de tomar cualquier decisión.

Deberá considerarse el diagnóstico de una reacción alérgica autoinmune por progesterona una vez que se hayan excluido desencadenantes extrínsecos de anafilaxis, así como de factores que simulen reacciones alérgicas multisistémicas.

El diagnóstico requiere una apropiada historia clínica acompañada de una prueba de inyección intradérmica de progesterona ${ }^{(21)}$. Algunos autores recomiendan realizar pruebas más extensas para evaluar de una mejor forma la evidencia inmunológica del proceso, mediante el envío de anticuerpos contra progesterona, prueba de activación de basófilos, inmunofluorescencia directa e indirecta a las células del cuerpo lúteo, liberación in vitro de interferón- $\gamma$ y anticuerpos circulantes contra la 17- $\alpha$-hidroxiprogesterona ${ }^{(22)}$. Debe mencionarse sin embargo, que en la mayoría de los reportes de casos en la literatura no se evalúa de forma rutinaria la evidencia serológica, ya que no siempre se ha encontrado que sea confiable.

Por lo tanto, las diferentes pruebas con preparaciones hormonales proveen la evidencia más clara de sensibilidad a hormonas. Existe un riesgo teórico de precipitar un evento anafiláctico severo por lo que se debe realizar con extrema precaución y por profesionales experimentados. El primer paso que se debe realizar es la prueba cutánea con progesterona acuosa, ya que las pruebas estimulantes pueden desencadenar una reacción alérgica severa. La progesterona acuosa por vía intramuscular es preferible al uso de Depo-Provera ${ }^{\circledR}$, la cual es una preparación de larga duración que contiene acetato de medroxiprogesterona y tiene el potencial de reacciones continuadas después de la inyección, además contiene un vehículo compuesto por polisorbato de polietilenglicol, metilparabeno, propilparabeno, cloruro de sodio y agua, el cual por sí mismo, podría ser una causa de una reacción cutánea o anafiláctica. Debido a lo anterior, se recomienda el uso de progesterona acuosa intramuscular para la prueba de sobrecarga en pacientes con posible sensibilidad a la progesterona en lugar de Depo-Provera ${ }^{\circledR}$.

Se considera una prueba positiva cuando la progesterona desencadena una reacción cutánea o sistémica después de su inyección intradérmica. Después de que la causa es identificada, se considera que no se justifican más estudios en aquellas pacientes que se logran controlar con agentes anovulatorios.

\section{Tratamiento}

La evidencia muestra que existen varios posibles tratamientos según los diferentes tipos de sensibilidad a la progesterona, por lo que debido a las diferencias en sintomatología se recomiendan regímenes individualizados, dependiendo de la manifestación de la sensibilidad a la progesterona. Cabe destacar que en algunas ocasiones la condición de la paciente podría resolver o remitir espontáneamente.

Las reacciones alérgicas autoinmunes por progesterona son usualmente resistentes a la terapia convencional con antihistamínicos. La utilización de glucocorticoides sistémicos, usualmente a altas dosis, en algunos estudios se ha reportado que controlan las lesiones cutáneas, por lo que pueden ser útiles en una exacerbación, sin embargo, su uso a largo plazo no se recomienda debido a sus efectos secundarios, además se debe tener en cuenta que su uso no ha logrado controlar las reacciones alérgicas en todos los $\operatorname{casos}^{(5)}$. 
Anteriormente, se utilizaba la desensibilización con progesterona como método de control para generar remisión de la enfermedad en la mayoría de las pacientes, pero se observó que 1 a 2 años después de suspender las inyecciones, algunas pacientes presentaban recurrencia de la enfermedad, por lo que en los últimos 10 años este tratamiento está cada vez más en desuso.

Igualmente, en el pasado, los estrógenos conjugados parecían ser la solución más simple, la mayoría de las pacientes presentaban mejoría al ser tratadas con dosis altas, hasta que se conoció la asociación de su uso con la potencial transformación maligna en el tejido endometrial, al no tener el efecto estabilizador de la progesterona.

Las modalidades terapéuticas actuales y la meta última del tratamiento es inhibir la secreción de progesterona endógena mediante la supresión de la ovulación ${ }^{(23)}$, ya que esto previene la producción endógena de progesterona, que es producida únicamente en ciclos ovulatorios.

Los esteroides 17- $\alpha$-alquilados como el Danazol, han sido utilizados para suprimir la ovulación en casos dermatológicos sencillos. Algunas pacientes responden con un aparente efecto sinérgico cuando se usan con una terapia crónica de corticosteroides a bajas dosis. Esta terapia puede traer complicaciones como elevación de transaminasas y/o lesión hepática ${ }^{(24)}$. Otros autores plantean el tratamiento profiláctico con Danazol en casos severos y resistentes, prescribiéndolo antes de la fecha probable de comienzo de la menstruación y continuándolo hasta 3 días después de terminar la menstruación, de esta forma, no se interfiere con el ciclo menstrual y simultáneamente se evitan los efectos adversos de la terapia a largo plazo con Danazol $^{(25)}$.

Otro medicamento utilizado es el Tamoxifeno, con resultados positivos y remisión de la sintomatología ${ }^{(26)}$. Los efectos secundarios más frecuentes relacionados a este medicamento incluyen la amenorrea y sus efectos negativos en el metabolismo del hueso.

Se han utilizados análogos de la GnRH con remisión de los síntomas, siendo la amenorrea el principal efecto secundario. La parte negativa de la terapia con GnRH es su alto costo y el hecho de que frecuentemente, se necesita suplementación con estrógenos para evitar los síntomas menopaúsicos como "calores", resequedad vaginal, pérdida de la libido y pérdida de la mineralización ósea ${ }^{(27)}$.

Los análogos de la LHRH se han utilizado también con gran éxito, aunque en algunos reportes de casos se descontinúan, ya que producen episodios de hipotensión que llegan a necesitar tratamiento con epinefrina $\mathrm{y}$ corticosteroides. Esto es frecuentemente observado ya que las dosis iniciales de LHRH primeramente estimulan los ovarios, lo que puede causar una exacerbación de los síntomas y $\mathrm{su}$ efecto en la supresión de la ovulación es a largo plazo.

En los casos con sintomatología severa o refractaria, se debe considerar la histerectomía con ooferectomía bilateral, la cual constituye el tratamiento definitivo ${ }^{(28)}$.

\section{CONCLUSIONES Y RECOMENDACIONES}

Las pacientes con reacciones de hipersensibilidad por progesterona se pueden presentar con diversas manifestaciones inusuales lo que provoca un retraso en el diagnóstico.

A pesar de que no se conoce con exactitud el mecanismo que está involucrado en la causa de la anafilaxis cíclica, ya sea la hipersensibilidad a la progesterona o a las prostaglandinas, la cesación del periodo menstrual e inducción de la menopausia a través de métodos médicos o quirúrgicos tiene como resultado el control de las reacciones anafilácticas.

La inducción de menopausia quirúrgica generalmente se reserva para las pacientes en las cuales falló el tratamiento médico debido a reacciones presentadas aún con adhesión al tratamiento o por efectos secundarios intolerables del mismo.

Es importante sospechar el diagnóstico de dermatitis autoinmune hormonal en las mujeres que se presenten con erupciones cutáneas que aparecen y desaparecen en relación con el ciclo menstrual. Sin embargo, la gran cantidad de resultados variables a la medicación supresora en muchos de los casos estudiados en la literatura, sugieren que es un desorden heterogéneo en el 
que múltiples mecanismos y mediadores juegan un papel importante.

\section{REFERENCIAS BIBLIOGRÁFICAS}

1. Meggs WJ Pescovitz OH Metacalfe D Loriaux DL Cutler G Kaliner, M. Progesterone sensitivity as a cause of recurrent anaphylaxis. N Engl J Med. 1984; 311:1236-1238.

2. Burstein M Rubinow A Shalit M. Cyclic anaphylaxis associated with menstruation. Ann Allergy. 1991;66:36-38.

3. Boggess KA Williamson HO Homm RJ. Influence of the menstrual cycle on systemic diseases. Obstet Gynecol Clin North Am. 1990; 17:321-342.

4. Riebel FA. Allergic coryza at menstruation from ovarian hormone. Ann Intern Med. 1935;9:91-92.

5. Snyder JL Krishnaswamy G. Autoimmune progesterone dermatitis and its manifestation as anaphylaxis: a case report and literature review. Ann Allergy Asthma Immunol 2003;90:469-477.

6. Herzberg AJ Strohmeryer CR CirilloHyland VA. Autoimmune progesterone dermatitis. J Am Acad Dermatol 1995; 32:335-338.

7. Geber H. Einege Daten zur Pathologie der Urticaria menstruationalis. Dermat Z. 1921;32:143.

8. Geber J. Desensitization in the treatment of menstrual intoxication and other allergic symptoms. $\mathrm{Br} \mathrm{J}$ Dermatol 1930;51:265-268.

9. Farah FS Shbaklu Z. Autoimmune progesterone urticaria. J Allergy Clin Immunol 1971;48:257-261.

10. Katayama I Nishioka K. Autoimmune progesterone dermatitis with persistent amenorrhoea. Br J Dermatol 1985;112: 487-491.

11. Osakay $\mathrm{T}$ Kutluay L Kaptanoglu A Karabacak O. Autoimmune progesterone dermatitis. Eur J Dermatol 2002; 6:589-591.

12. Mutasim DF Baumbach JL. Bullous autoimmune estrogen dermatitis. J Am Acad Dermatol 2003;49:130-131.
13. Meggs WJ Pescovitz OH Metacalfe D Loriaux DL Cutler G Kaliner M. Progesterone sensitivity as a cause of recurrent anaphylaxis. N Engl J Med. 1984;311:1236-1238.

14. Slater JE Raphael G Cutler GB Jr. Loriaux DL Meggs WJ Kaliner M. Recurrent Anaphylaxis in Menstruating Women: Treatment With a Luteinizing Hormone-Releasing Hormone Agonist- a preliminary report. Obstet Gynecol. 1987;70:542-546.

15. Tauber AI Kaliner M Stechschulte DJ Austen KF. Immunologic release of histamine and slow reacting substance of anaphylaxis from human lung. J Immunol. 1973;111:27-32.

16. Basomba A Guerrero M Campos A Pelaz A Villalmanzo IG. Grave anaphylactic-like reaction in the course of menstruation. Allergy. 1987;42:477479.

17. Bernstein IL Bernstein DI Lummus ZL Bernstein JA. A case of progesteroneinduced anaphylaxis, cyclic urticaria/angioedema, and autoinmune dermatitis. J Womens Health. 2011;20(4):643648.

18. Slater JE Kaliner M. Effects of sex hormones on basophil histamine release in recurrent idiopathic anaphylaxis. J Allergy Clin Immunol. 1987;80:285-290.

19. Metcalfe DD. Differential diagnosis of the patient with unexplained flushing/anaphylaxis. Allergy Asthma Proc. 2000;21:21-24.

20. Szczeklik A. Adverse reactions to aspirin and nonsteroidal anti-inflammatory drugs. Ann Allergy. 1987;59:113-118.

21. Stranahan D RauschD Deng A Gaspari A. The role of intradermal skin testing and patch testing in the diagnosis of autoimmune progesterone dermatitis. Dermatitis 2006;17(1):39-42.

22. Halevy $\mathrm{S}$ Cohen AD Lunenfeld E Grossman N. Autoimmune progesterone dermatitis manifested as erythema annulare centrifugum: Confirmation of progesterone sensitivity by in vitro interferon-gamma release. J Am Acad Dermatol 2002;47:311-313. 
23. Teelucksingh S Edwards CR. Autoimmune progesterone dermatitis. J Intern Med 1990;227:143-144.

24. Brestel EP Thrush LB. The treatment of glucocorticoid dependent chronic urticaria with stanozolol. J Allergy Clin Immunol. 1988;82:265-269.

25. Shahar E Bergman R Pollack S. Autoimmune progesterone dermatitis: effective prophylactic treatment with danazol. Int J Dermatol. 1997;36:708711.

26. Stephens CJ Wojnarowska FT Wilkinson JD. Autoimmune progesterone dermatitis responding to tamoxifen. $\mathrm{Br} \mathrm{J}$ Dermatol. 1989;121:135-137.

27. Rodenas JM Herranz MT Tercedor J. Autoimmune progesterone dermatitis: treatment with oophorectomy. $\mathrm{Br} \mathrm{J}$ Dermatol. 1998;139:508-511.

28. Shelley WB Preucell RW Spoont SS. Autoimmune progesterone dermatitis. Cure by oophorectomy. JAMA 1964; 190:35-38.

29. Simpson G Roomes D Humphrey MD. Anaphylactoid reactions associated with menstruation affecting two sisters. Med J Aust. 2001;175:415-417.

\section{DECLARACIÓN DE CONFLICTO DE IN- TERESES}

Los autores declaran que no existen conflictos de intereses. 